\section{Prevalência e fatores associados à ocorrência de anemia entre menores de seis anos de idade em Pelotas, RS}

\section{Prevalence of anemia and associated factors among children under six years of age in Pelotas, South Brazil}

\author{
Iná dos Santos \\ Programa de Pós-graduação em Epidemiologia \\ Centro de Pesquisas Epidemiológicas \\ Universidade Federal de Pelotas \\ Av. Duque de Caxias, 250 - $3^{\circ}$ piso, CP 464 \\ 96001-970 - Pelotas, RS \\ inasantos@uol.com.br
}

Juraci Almeida César

Departamento Materno-Infantil

Universidade Federal do Rio Grande, RS

Gicele Minten

Programa de Pós-graduação em Epidemiologia

Centro de Pesquisas Epidemiológicas

Universidade Federal de Pelotas

\section{Neiva Valle}

Programa de Pós-graduação em Epidemiologia

Centro de Pesquisas Epidemiológicas

Universidade Federal de Pelotas

\section{Nelson Arns Neumann}

Coordenação Nacional da Pastroal da Criança, Curitiba, PR

\section{Eduardo Cercato}

Departamento Materno-Infantil

Universidade Federal do Rio Grande, RS

Estudo financiado pela Fundação Itaú Social através da Pastoral da Criança e realizado no Programa de Pós-graduação em Epidemiologia do Centro de Pesquisas Epidemiológicas da Universidade Federal de Pelotas.

\section{Resumo}

Objetivos: Determinar a prevalência e investigar fatores associados à ocorrência de anemia entre menores de seis anos de idade atendidos pela Pastoral da Criança em Pelotas, RS. Métodos: Na fase de rastreamento de uma intervenção nutricional, as crianças foram avaliadas através de estudo transversal. No domicílio, após consentimento informado, a mãe foi entrevistada, sendo coletadas informações sobre características demográficas e socioeconômicas da família, saúde da criança, características ao nascer, amamentação e freqüência semanal de consumo de alimentos. As crianças foram pesadas e medidas. Foi coletada uma amostra de sangue capilar da polpa digital em microcuveta e a leitura da concentração de hemoglobina foi feita em fotômetro portátil (Hemocue). Considerou-se como tendo anemia quando a concentração de hemoglobina era inferior a $11 \mathrm{~g} / \mathrm{dl}$. Por se tratar de desfecho freqüente, a associação com as variáveis independentes foi analisada por regressão de Poisson. A análise obedeceu a modelo hierárquico previamente definido. Resultados: Foram identificadas 362 crianças menores de seis anos, sendo a hemoglobina dosada em 304 (84,0\%) delas. A prevalência de anemia foi de 53,0\% (IC95\% 47,2\% $58,7 \%)$. Os fatores de risco foram a menor idade da criança, cor não branca, presença de família numerosa e classe social E (em comparação à D). A disponibilidade de água encanada dentro de casa e o maior peso ao nascer mostraram-se fatores protetores. Conclusões: os achados deste estudo mostram uma alta prevalência de anemia entre as crianças alvo da ação da Pastoral da Criança, bem como a presença de diversos fatores de risco e poucos fatores de proteção, o que mostra a necessidade de intervenções preventivas.

Palavras-chave: Deficiência de ferro. Anemia ferropriva. Fatores de risco. Pastoral da Criança. 
Abstract

Objectives: to determine the prevalence of and investigate factors associated with anemia among children under six years of age, assisted by the Pastoral da Criança in Pelotas, South Brazil. Methods: during the screening phase of a community intervention, selected children were studied through a crosssectional approach. At the household level, after informed consent, mothers were interviewed on demographic and socio-economic characteristics of the family, child health, characteristics at birth, breast-feeding, and weekly frequency of food consumption. Children were weighed and had their height measured. A portable Hemocue photometer was used for determining the hemoglobin level in capillary blood samples. Anemia was defined as hemoglobin concentration below 11g/dl. Because of the high prevalence of anemia, analyses of association with independent variables were carried out through Poisson regression. Adjusted analyses followed a hierarchical model previously defined. Results: Among 362 selected children, hemoglobin was determined for $304(84.0 \%)$. The prevalence of anemia was $53.0 \%$ (95\%CI $47.2 \%-58.7 \%$ ). Risk factors for anemia were: young age, non-white skin color, large family, and low social class (E compared to D). Higher weight at birth and availability of drinking water at the household level were protective factors for anemia. Conclusion: the findings of this study show that the prevalence of anemia is high among the Pastoral da Criança target population, with a large number of risk factors and some protective factors indicating an urgent need for preventive interventions.

Key Words: Iron deficiency. Iron-deficiency anemia. Risk factors. Pastoral da Criança.

\section{Introdução}

No Brasil, estudos revelam aumento da prevalência de anemia na infância ao longo dos anos, ${ }^{5,9}$. Dados recentes mostram prevalências no país que variam de $26,7 \%$ a $60,4 \%^{3,8-10,15,16}$.

Três fatores contribuem para a alta prevalência de anemia na infância: as reservas de ferro ao nascer (menores entre os prematuros e os nascidos com baixo peso), a velocidade de crescimento (no primeiro ano de vida, a criança nascida a termo triplica seu peso de nascimento) e o balanço entre a ingestão e as perdas do mineral. O aleitamento materno nos primeiros seis meses de vida, especialmente se for exclusivo, constitui-se em importante fator de proteção contra a anemia. Apesar do baixo conteúdo em ferro $(0,26$ a $0,73 \mathrm{mg} / \mathrm{ml})$, a biodisponibilidade do mineral é alta no leite humano (taxa de absorção de $48,0 \pm 25,5 \%$, contra $19,5 \pm 17,3 \%$ a partir do leite de vaca $)^{4}$.Após os seis meses de idade, a composição da dieta é o mais importante fator para a manutenção de um adequado estado nutricional de ferro. O baixo consumo de ferro e sua baixa biodisponibilidade nos alimentos que compõem a dieta são a principal causa de anemia nainfância.

A biodisponibilidade do ferro depende do tipo de alimento consumido e da combinação desse com outros na dieta. $\mathrm{O}$ ferro heme, derivado da hemoglobina e da mioglobina e presente na carne de gado bovino, peixes, aves, vísceras e embutidos, apresenta alta biodisponibilidade, sendo absorvida pela mucosa intestinal 10 a $30 \%$ da quantidade consumida. $\mathrm{O}$ ferro não heme, derivado dos produtos vegetais (cereais, leguminosas e tubérculos) tem biodisponibilidade variável, geralmente baixa. Sua biodisponibilidade é potencializada pelo consumo concomitante de carnes, vísceras e alimentos ricos em ácidos orgânicos, como o ácido ascórbico, vitamina A e beta carotenos.

O objetivo desse estudo foi determinar a prevalência e investigar fatores associados à ocorrência de anemia entre menores de seis anos de idade em população alvo da ação da Pastoral da Criança em Pelotas, RS. 


\section{Métodos}

Os dados apresentados são parte da primeira fase de um estudo experimental, tipo intervenção comunitária, realizada nas comunidades atendidas pela Pastoral da Criança na periferia de Pelotas $^{12}$. A população em estudo foi constituída de crianças menores de seis anos residentes nos bairros Getúlio Vargas, Pestano, Santa Terezinha, Sítio Floresta e vilas Farroupilha e Princesa, que vinham sendo cobertos pela Pastoral da Criança. Todas as crianças cujos nomes apareciam no Caderno da Líder e que, portanto, eram visitadas mensalmente pelas líderes da Pastoral da Criança foram incluídas neste estudo. Estas crianças foram posteriormente visitadas em seus domicílios por entrevistadores previamente treinados e por um profissional que fazia a leitura do nível de hemoglobina através de fotômetro portátil conforme descrito abaixo em maiores detalhes.

O cálculo da precisão da estimativa de prevalência de anemia, bem como o poder da amostra em detectar associações foi feito a posteriori. Com uma amostra de 304 crianças (dentre as 362 das quais foi possível coletar sangue para dosagem de hemoglobina), o estudo era capaz de detectar a prevalência observada de $53,0 \%$, com erro inferior a 0,2 pontos percentuais, significativa em nível de 95\%. Para as medidas de associação, a amostra tinha um poder de $80 \%$ de detectar riscos iguais ou superiores a 1,4, significativos em nível de 95\%, expandida em $10 \%$ para controlar possível efeito de fatores de confusão, para exposições com prevalência de 25 a $75 \%$, assumindo-se uma prevalência de anemia de $49 \%$ entre os não expostos.

O trabalho de campo foi realizado por quatro entrevistadoras previamente treinadas que visitavam a criança no domicílio. Após consentimento informado, a mãe era entrevistada e a criança examinada. Em cada residência, eram incluídas todas as crianças da faixa etária do estudo, desde que atendidas pela Pastoral. Um questionário estruturado e pré-codificado foi utilizado para a entrevista com a mãe ou, na ausência desta, com o responsável pela criança. As mães foram inquiridas quanto a características demográficas e socioeconômicas da família (idade da mãe; escolaridade da mãe em anos completos; trabalho materno remunerado atual; pai ou padrasto vivendo no mesmo domicílio; escolaridade do pai ou padrasto em anos completos; situação atual de trabalho do pai ou padrasto; total de moradores do domicílio; número de menores de seis anos de idade no domicílio, incluindo a criança do estudo; número de avós vivendo no mesmo domicílio; número de pessoas por quarto de dormir; renda familiar mensal; classe social obtida pelo escore da Associação Nacional de Empresas de Pesquisa (ANEP), compreendendo a escolaridade da pessoa com maior renda do domicílio; número de rádios disponíveis no domicílio; posse de geladeira, máquina de lavar roupas e televisão em cores; e disponibilidade de água encanada dentro de casa e de sanitário com descarga. Com base nestas variáveis, a ANEP criou escores que variam de 0 (pior) a 34 (melhor), e este escore foi transformado em categorias que correspondem a classe social. Famílias com escore entre 0 e 5 pertencem à classe $\mathrm{E}$, entre 6 e 10 à classe $\mathrm{D}$, entre 11 e 16 à classe $\mathrm{C}$, entre $17 \mathrm{e}$ 20 à classe B2, entre 21 e 24 à classe B1, entre 25 e 29 à classe $A 2$ e, os mais abastados, com escore de 30 a 34, são classificados como de classe A. Informações mais detalhadas sobre esta classificação podem ser obtidas em www.anep.org.br.

Sobre a criança investigaram-se as características demográficas (sexo, cor da pele e idade em meses completos), morbidades (diarréia no dia da entrevista; diarréia nos últimos 15 dias; tosse com ou sem febre, dispnéia ou congestão nasal, nos últimos sete dias; número de hospitalizações nos 12 meses anteriores a entrevista; diagnóstico atual ou passado de anemia feito por médico; uso atual de medicamentos; verificação da embalagem dos medicamentos em uso, para checar consumo atual de sais de ferro; e estado vacinal de acordo com a idade), hábitos alimentares (amamentação atual entre os menores de três anos de idade; idade de desmame; consumo semanal de carne, fígado de ave, gema de ovo, multimistura e, en- 
tre os menores de oito meses de idade, de papa grossa; além de adição de uma colher das de chá de óleo, manteiga ou margarina, ao prato da criança), características ao nascer (prematuridade, peso e comprimento) e estado nutricional atual para os indicadores peso para a idade, comprimento/altura para idade e peso para altura, tendo como referência a população do National Center of Health Statistics - NCHS).

A variável dependente (concentração de hemoglobina em $\mathrm{g} / \mathrm{dl}$ ) foi obtida através da leitura direta em fotômetro portátil (hemoglobinômetro HemoCue) de amostra de sangue capilar da polpa digital colhido em microcuvetas. Considerou-se anemia a concentração de hemoglobina inferior a $11 \mathrm{~g} /$ dl, conforme recomendação da OMS².

Sobre a Líder da Pastoral, investigou-se se havia visitado a criança no último mês; se, na última visita, entrou na casa, viu a criança, aconselhou a mãe sobre alimentação e convidou para o Dia da Celebração da Vida (DCV); e, havendo a mãe comparecido às atividades do DCV, se a criança foi pesada, se a Líder falou sobre saúde, se a criança recebeu lanche e se a mãe ficou até o final da pesagem.

Por ser alta a prevalência de anemia na amostra estudada, a força da associação, tanto nas análises bruta e ajustada, entre as variáveis independentes e o desfecho, foi aferida mediante regressão de Poisson com estimativa robusta. Esta técnica evita eventuais superestimativas dos riscos observados. Na análise multivariada utilizou-se a função "cluster", tendo como variável definidora do aglomerado o bairro de residência da criança. A seleção das variáveis para o modelo final foi feita pelo método retrógrado. O programa Stata 6.0 foi utilizado para as análises de regressão.

A análise multivariada seguiu um modelo hierárquico, sendo considerados quatro níveis de determinação da anemia. O primeiro nível foi representado pelas características socioeconômicas e demográficas da família; o segundo, pelas características da criança ao nascer; o terceiro, pelas características demográficas, a saúde e a alimenta- ção da criança, além das variáveis de atuação da Líder, que lidam basicamente com a dieta da criança e com o manejo de doenças comuns, daí a sua localização neste nível; e, no quarto nível, o desfecho (prevalência de anemia). Foram levadas para a análise multivariada as variáveis consideradas potenciais fatores de confusão, ou seja, as que se mostraram associadas à prevalência de anemia ao nível de $\mathrm{p}<0,20$.

O protocolo do estudo foi aprovado pelo Comitê de Ética da Faculdade de Medicina da Universidade Federal de Pelotas. Cada criança só foi incluída no estudo após a obtenção do consentimento informado e por escrito da mãe ou responsável. As crianças diagnosticadas como anêmicas (hemoglobina $<11 \mathrm{~g} / \mathrm{dl}$ ) foram encaminhadas para o posto de saúde mais próximo de sua residência.

\section{Resultados}

Dentre as 362 crianças incluídas no estudo obteve-se amostra de sangue para dosagem de hemoglobina de 304 delas, o que representa $16 \%$ de perdas. As perdas foram um pouco maiores nas famílias com apenas uma criança $(21,0 \%)$, comparativamente às com duas $(14,6 \%)$ ou mais $(8,0 \%)(p=0,04)$. O principal motivo das perdas foi não encontrar a mãe e a criança no domicílio, após três tentativas, em dias e horários diferentes. A prevalência de anemia entre as crianças estudadas foi de 53,0\% (IC95\% 47,2\% $58,7 \%)$. A Tabela 1 mostra a distribuição da amostra, a prevalência de anemia e as razões de prevalências brutas, conforme características demográficas e ao nascer da amostra. Houve associação estatisticamente significativa com a cor da pele: $64,0 \%$ das crianças não brancas contra 49,6\% das brancas apresentavam anemia, o que representa um risco $29 \%$ maior $(p=0,02)$. A prevalência de anemia variou inversamente com a idade. Entre os menores de 12 meses de idade, a prevalência foi de $81,1 \%$, decrescendo para $75,5 \%$ entre as crianças de 12 a 23,9 meses, para $60,3 \%$ no terceiro ano de vida, $41,5 \%$ no quarto ano, $40,4 \%$ no quinto e $25,6 \%$ no sex- 
to (p $<0,001)$. O risco bruto de anemia foi 2,17 vezes maior no primeiro ano de vida, comparativamente às crianças de cinco anos, tomadas como referência.

Exceto a prematuridade e o comprimento, os demais indicadores ao nascer associaram-se significativamente com o risco bruto de anemia (Tabela 1). As crianças no quartil mais baixo de peso ao nascer apresentaram a maior prevalência de anemia (63,5\%), contra $39,6 \%$ no quartil mais elevado, sendo a associação linear inversa, com menores riscos entre os nascidos com maior peso. Entre as crianças nascidas com baixo peso ( $<2500$ gramas), a prevalência foi de $71,9 \%$, comparativamente a $50,7 \%$ entre as controles. Nascer com baixo peso implicou aumento bruto de risco de $42 \%$ ( $p=0,006$ ).

A idade materna mostrou-se associada de forma inversa e significativa à prevalência de anemia nas crianças (Tabela 2a). A prevalência foi maior em filhos de mães mais jovens, em comparação com os filhos de mães mais velhas. $\mathrm{O}$ trabalho materno fora de casa e a escolaridade da mãe não mostraram associação com a ocorrência de anemia.

As crianças cujo pai ou padrasto residia no domicílio apresentaram prevalência menor de anemia ( $49,8 \%$ versus $67,8 \%$ ). Houve associação linear e inversa entre a escolaridade do pai ou padrasto e a prevalência de anemia, sendo esta menor entre os filhos daqueles que possuíam entre 5 e 13 anos de educação formal $(37,2 \%)$, comparativamente aos filhos de não alfabetizados (52,0\%) ou com menos de cinco anos de escola $(61,7 \%)$. Os filhos de pais/padrastos com 5-13 anos de escolaridade apresentaram um risco bruto de anemia $29 \%$ menor do que o dos filhos de analfabetos.

Não mostraram associação estatisticamente significativa com a prevalência de anemia as seguintes variáveis: número total de moradores e de menores de seis anos de idade no domicílio, presença de avós vivendo no domicílio e número de pessoas por quarto.

A Tabela $2 b$ mostra que as crianças de famílias da classe social E apresentaram uma prevalência de anemia de $57,7 \%$ contra $38,9 \%$ entre as crianças da classe social D. Esta diferença foi estatisticamente significativa e representa um risco cerca de $50 \%$ maior. A associação com a renda familiar foi linear e inversa: entre as crianças de famílias do menor quartil de renda a prevalência foi de $66,2 \%$, decrescendo para $55,7 \%, 46,3 \%$ e $42,6 \%$, respectivamente, no segundo, terceiro e quarto quartis. Quanto maior a renda familiar, menor o risco de a criança apresentar anemia $(p=0,03)$. As associações com abastecimento de água no domicílio e com o tipo de destino dado, no domicílio, aos dejetos humanos foram também significativas, sendo o risco $41 \%$ e $34 \%$ menores, respectivamente, entre crianças de residências com melhores condições sanitárias.

Na Tabela 3, as crianças que apresentaram diarréia nas duas semanas anteriores à entrevista tiveram uma prevalência maior de anemia $(70,0 \%)$ em relação àquelas sem história de diarréia (51,0\%), o que representa um risco $27 \%$ maior $(p=0,02)$. Entre as crianças com estado vacinal incompleto para a idade, o risco de anemia foi $27 \%$ maior, comparativamente às demais. Quanto às demais morbidades e aos outros aspectos da saúde da criança investigados, não se observou associação estatisticamente significativa com a ocorrência ou o risco bruto de anemia.

Quanto à amamentação e aos hábitos alimentares (Tabela 4), observou-se menor prevalência de anemia (20,0\%) entre as crianças que consumiam multimistura semanalmente do que entre as demais $(55,1 \%)$, sendo o risco bruto $64 \%$ menor entre as primeiras. Entre os menores de oito meses de idade, o risco foi $87 \%$ maior entre aqueles que recebiam papa grossa quando comparadas às demais $(\mathrm{p}=0,03)$.

Não houve associação bruta estatisticamente significativa entre o risco de anemia e os indicadores nutricionais estudados (Tabela 5). Da mesma forma, não se observou associação entre os aspectos estudados do desempenho da Líder e prevalência ou o risco de anemia.

Todas as variáveis associadas ao desfecho em um nível de significância menor do que 0,20 foram levadas para a análise 
Tabela 1 - Prevalência de anemia e razão de prevalências bruta (com intervalo de confiança de 95\%), conforme características demográficas, idade gestacional e antropometria para crianças menores de seis anos. Pastoral da Criança, Pelotas, RS, 2003.

Table 1 - [Prevalence of anemia and overall prevalence rates (with 95\% confidence interval) according to demographic characteristics, gestational age and anthropometry for children under six years of age. Pastoral da Criança, Pelotas, RS, 2003.]

\begin{tabular}{|c|c|c|c|c|}
\hline Característica & $N(304)$ & $\begin{array}{l}\text { Prevalência } \\
(\%)\end{array}$ & $\begin{array}{c}\text { Razão de prevalência } \\
\text { bruta e IC95\% }\end{array}$ & P-valor \\
\hline \multicolumn{5}{|l|}{ Sexo } \\
\hline Masculino & 159 & 51,6 & 1,00 & 0,6 \\
\hline Feminino & 145 & 54,5 & $1,06(0,85-1,31)$ & \\
\hline \multicolumn{5}{|l|}{ Cor } \\
\hline Branca & 228 & 49,6 & 1,00 & 0,02 \\
\hline Não branca & 75 & 64,0 & $1,29(1,04-1,60)$ & \\
\hline \multicolumn{5}{|l|}{ Idade em meses } \\
\hline$<12$ & 37 & 81,1 & $3,17(1,86-5,41)$ & $<0,001$ \\
\hline $12-23,9$ & 49 & 75,5 & $2,95(1,73-5,04)$ & \\
\hline $24-35,9$ & 58 & 60,3 & $2,36(1,36-4,10)$ & \\
\hline $36-47,9$ & 65 & 41,5 & $1,62(0,90-2,92)$ & \\
\hline $48-59,9$ & 52 & 40,4 & $1,58(0,86-2,90)$ & \\
\hline $60-71,9$ & 43 & 25,6 & 1,00 & \\
\hline \multicolumn{5}{|l|}{ Prematuridade } \\
\hline Não & 267 & 51,3 & 1,00 & 0,1 \\
\hline Sim & 36 & 63,9 & $1,25(0,95-1,64)$ & \\
\hline \multicolumn{5}{|c|}{ Peso ao nascer ( $\mathrm{g}$ ) em quartis } \\
\hline $1260-2799$ & 63 & 63,5 & 1,00 & 0,02 \\
\hline $2800-3169$ & 68 & 55,9 & $0,88(0,66-1,17)$ & \\
\hline $3170-3499$ & 72 & 59,7 & $0,94(0,72-1,23)$ & \\
\hline $3500-4860$ & 101 & 39,6 & $0,62(0,46-0,85)$ & \\
\hline \multicolumn{5}{|c|}{ Baixo peso ao nascer } \\
\hline Não & 272 & 50,7 & 1,00 & 0,006 \\
\hline Sim & 32 & 71,9 & $1,42(1,11-1,81)$ & \\
\hline \multicolumn{5}{|c|}{ Comprimento $(\mathrm{cm})$ ao nascer (quartis) } \\
\hline $35,5-46,9$ & 60 & $61,7(37)$ & 1,00 & 0,2 \\
\hline $47,0-47,9$ & 34 & $50,0(17)$ & $0,81(0,55-1,20)$ & \\
\hline $48-49,9$ & 92 & $55,4(51)$ & $0,90(0,69-1,18)$ & \\
\hline$\geq 50,0$ & 86 & $43,0(37)$ & $0,70(0,51-0,96)$ & \\
\hline
\end{tabular}

multivariada, exceto o comprimento ao nascer, devido à forte correlação com o peso de nascimento, e a escolaridade do pai/companheiro, por estar incluída na variável de classe social. Inicialmente foram incluídas no modelo as variáveis idade, escolaridade e trabalho materno; presença do pai/companheiro; renda familiar; privada com descar- ga no domicílio; e família numerosa. Nessa análise, as crianças da classe social E apresentaram um risco $37 \%$ maior de serem anêmicas, quando comparadas com as da classe $\mathrm{D}(\mathrm{RP}=1,37$; IC95\% 1,20-1,58; $\mathrm{p}<0,001)$. As crianças que viviam em famílias numerosas apresentaram um risco $51 \%$ maior de serem anêmicas do que seus pares $(\mathrm{RP}=1,51$; 
Tabela 2a - Prevalência de anemia e razão de prevalências bruta (com intervalo de confiança de 95\%) conforme características demográficas maternas e condições socioeconômicas das famílias de crianças menores de seis anos. Pastoral da Criança, Pelotas, RS, 2003.

Table $\mathbf{2} \boldsymbol{a}$ - Prevalence of anemia and overall prevalence rates (with 95\% confidence interval) according to maternal demographic characteristics and socio economic status of the families of children under six years of age. Pastoral da Criança, Pelotas, RS, 2003.

\begin{tabular}{|c|c|c|c|}
\hline Característica & $\mathrm{N}(304)$ & $\begin{array}{l}\text { Prevalência } \\
\text { (\%) }\end{array}$ & $\begin{array}{c}\text { Razão de prevalência } \\
\text { bruta e IC95\% }\end{array}$ \\
\hline
\end{tabular}

Idade mãe (anos)

$\begin{array}{lccc}<20 & 22 & 72,7 & 1,00 \\ 20-29 & 142 & 42,9 & 0,79(0,59-1,06) \\ 30-39 & 112 & 53,6 & 0,59(0,42-0,82) \\ \geq 40 & 28 & & 0,74(0,48-1,13)\end{array}$

Escolaridade da mãe em anos completos

0

$1-4$

5-8

$9-11$

Mãe trabalhando fora

Não

Sim

Presença pai/padrasto

Não

Sim

Escolaridade pai em anos completos

0

1-4

5-13

Pai desempregado

Não

Sim

Crianças menores de seis anos no domicílio

1

2

3-5

Avó(s) morando junto

Não

Sim

Total moradores

2-4

5-7

8-14

$\geq 3$ pessoas/quarto

Não

Sim
112

147

45

86

218
59

243

201

45

124

111

69

264

40

21

24

856

37,2

49,8

48,9

50,0

50,0

56,5

51,1

65,0

50,0

56,5

48,9
1,00

$1,19(0,79-1,78)$

$0,71(0,46-1,11)$
0,1

$0,75(0,53-1,05)$

$0,69(0,48-0,98)$

$0,52(0,28-0,97)$

1,00

0,7

$1,05(0,81-1,37)$

1,00

0,005

$0,73(0,59-0,91)$

1,00

$1,02(0,73-1,42)$

0,9

$1,08(0,85-1,38)$

$1,13(0,86-1,48)$

0,002
52,3

3,2
1,00

$1,27(0,98-1,64)$

1,00

$1,23(0,89-1,43)$

$0,98(0,69-1,39)$
1,00

0,7

0,07

1,00

0,9 
Tabela $\mathbf{2 b}$ - Prevalência de anemia e razão de prevalências bruta (com intervalo de confiança de 95\%) conforme características demográficas maternas e condições sócio-econômicas das famílias de crianças menores de seis anos. Pastoral da Criança, Pelotas, RS, 2003.

Table $\mathbf{2} \boldsymbol{b}$ - Prevalence of anemia and overall prevalence rates (with 95\% confidence interval) according to maternal demographic characteristics and socio economic status of the families of children under six years of age. Pastoral da Criança, Pelotas, RS, 2003.

\begin{tabular}{|c|c|c|c|c|}
\hline Característica & $\mathrm{N}(304)$ & $\begin{array}{l}\text { Prevalência } \\
\text { (\%) }\end{array}$ & $\begin{array}{l}\text { Razão de prevalência } \\
\text { bruta e IC95\% }\end{array}$ & P-valor \\
\hline \multicolumn{5}{|l|}{ Classe social } \\
\hline$D$ & 108 & 38,9 & 1,00 & 0,005 \\
\hline E & 137 & 57,7 & $1,48(1,12-1,96)$ & \\
\hline \multicolumn{5}{|l|}{ Renda (R\$)(quartis) } \\
\hline $0,00-150,00$ & 68 & 66,2 & 1,00 & 0,03 \\
\hline $151,00-240,00$ & 70 & 55,7 & $0,84(0,64-1,10)$ & \\
\hline $241,00-375,00$ & 67 & 46,3 & $0,70(0,51-0,95)$ & \\
\hline $376,00-1750,00$ & 61 & 42,6 & $0,64(0,46-0,90)$ & \\
\hline \multicolumn{5}{|l|}{ Água encanada } \\
\hline Não & 31 & 83,9 & 1,00 & $<0,001$ \\
\hline Sim & 266 & 49,2 & $0,59(0,48-0,72)$ & \\
\hline \multicolumn{5}{|l|}{ Privada c/descarga } \\
\hline Não & 52 & 73,1 & 1,00 & $<0,001$ \\
\hline Sim & 230 & 48,3 & $0,66(0,53-0,82)$ & \\
\hline
\end{tabular}

IC95\% $1,12-2,04 ; \mathrm{p}=0,007)$. Entre as crianças que viviam em domicílios com água encanada dentro de casa, o risco de serem anêmicas foi $40 \%$ menor do que o observado entre as demais $(\mathrm{RP}=0,60$; IC95\% 0,460,$78 ; \mathrm{p}<0,001$ ) (Tabela 6).

Das variáveis do segundo nível (características da criança ao nascer), apenas o peso ao nascer manteve-se significante após ajuste para as variáveis do primeiro. Entre as crianças cujo peso ao nascer pertencia ao quartil superior da distribuição da amostra, o risco foi $42 \%$ menor do que o observado entre as do quartil mais inferior $(\mathrm{RP}=0,58$; IC95\% $0,43-0,79 ; \mathrm{p}<0,001$ ).

No terceiro nível, ajustado para os demais, somente a idade e a cor da pele mantiveram-se associadas ao desfecho. As crianças de pele não branca apresentaram um risco $32 \%$ maior de ter anemia em relação às de cor branca (RP = 1,32; IC95\% 1,02-1,69; $p$ $=0,03)$. O risco diminuiu com a idade $(\mathrm{p}<$ $0,001)$, sendo duas a três vezes maior entre os menores de três anos quando compara- dos aos de cinco anos de idade. Entre os menores de 12 meses, a RP foi 3,36 vezes maior do que o observado entre as crianças de cinco anos de idade. Todas estas razões de prevalências ajustadas estão mostradas na Tabela 6.

\section{Discussão}

A prevalência observada de anemia (53,0\%; IC95\% 47,2\% - 58,7\%) é maior do que a encontrada por outros estudos entre crianças brasileiras. Monteiro e Szarfarc ${ }^{5}$, estudando 912 menores de cinco anos do município de São Paulo, encontraram uma prevalência de 35,6\% (IC95\% 32,5\% - 38,9\%). Osório et al. ${ }^{10}$ detectaram prevalência de 40,9\% entre 777 crianças com idade entre $6 \mathrm{e}$ 59 meses no estado de Pernambuco, (IC95\% $37,5 \%$ - 44,5\%). Entre 1287 pré-escolares residentes em zonas urbanas do estado da Paraíba, Oliveira et al. ${ }^{9}$ encontraram prevalência de anemia de 36,4\% (IC95\% 33,7\% $39,1 \%)$. Tendo em vista que a prevalência 
Tabela 3 - Prevalência de anemia e Razão de Prevalências Bruta (com intervalo de confiança de $95 \%$ ) conforme morbidades comuns na infância e estado vacinal das crianças. Pastoral da Criança, Pelotas, RS, 2003.

Table 3 - Prevalence of anemia and overall prevalence rates (with 95\% confidence interval) according to common childhood diseases and vaccination status of children. Pastoral da Criança, Pelotas, RS, 2003.

\begin{tabular}{|c|c|c|c|}
\hline Característica & $N(304)$ & $\begin{array}{l}\text { Prevalência } \\
\text { (\%) }\end{array}$ & $\begin{array}{c}\text { Razão de prevalência } \\
\text { bruta e IC95\% }\end{array}$ \\
\hline
\end{tabular}

Diarréia nas últimas 24 horas

Não

Sim

Diarréia nos últimos 15 dias

Não

Sim

Tosse nos últimos sete dias

Não

Sim

164

140

107

33

Sim

Tosse+dispnéia

Sim

Não

Tosse+congestão nasal

Não

Sim

Hospitalizações

Não

Sim

172

132

134

163

Não

Sim

Anemia relatada

Não

Sim

22

Medicamentos

Não

Sim
52,9

53,0

49,3

51,7

50,0

1,00

$0,97(0,63-1,49)$

52,2

72,7

51,0

70,0

51,8

54,3

53,3

57,6

54,5

54,0

51,7

56,3

1,00

1,00

$1,00(0,81-1,24)$

1,00

$1,13(0,90-1,41)$

0,3

55,8

50,8

62,1

1,00

$1,22(0,96-1,55)$

64,0

57,6

1,00

0,7

0,7

0,9

0,6
$0,92(0,67-1,26)$
1,00

$0,90(0,59-1,37)$
0,09

0,02

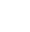

(1)

Sulfato ferroso'

Não

$\begin{array}{ll}25 & 64,0 \\ 33 & 57,6\end{array}$

69,4

1,00

0,01

Não

'somente para crianças que estavam usando algum medicamento / 'only for children who were using medication 
Tabela 4 - Prevalência de anemia e razão de prevalências Bruta (com intervalo de confiança de 95\%) conforme práticas alimentares da criança. Pastoral da Criança, Pelotas, RS, 2003.

Table 4 - Prevalence of anemia and overall prevalence rates (with 95\% confidence interval) according to children's diet. Pastoral da Criança, Pelotas, RS, 2003.

\begin{tabular}{|c|c|c|c|c|}
\hline Característica & $N(304)$ & $\begin{array}{l}\text { Prevalência } \\
\text { (\%) }\end{array}$ & $\begin{array}{l}\text { Razão de prevalência } \\
\text { bruta e IC95\% }\end{array}$ & P-valor \\
\hline \multicolumn{5}{|c|}{ Se estava mamando ${ }^{1}$} \\
\hline Não & 131 & 61,8 & 1,00 & 0,3 \\
\hline Sim & 40 & 70,0 & $1,13(0,89-1,45)$ & \\
\hline \multicolumn{5}{|c|}{ Foi amamentado ${ }^{2}$} \\
\hline Não & 18 & 44,4 & 1,00 & 0,6 \\
\hline Sim & 245 & 50,6 & $1,14(0,67-1,94)$ & \\
\hline \multicolumn{5}{|c|}{ Consumo semanal Carne } \\
\hline Não & 53 & 60,4 & 1,00 & 0,2 \\
\hline Sim & 247 & 51,0 & $0,84(0,66-1,09)$ & \\
\hline \multicolumn{5}{|l|}{ Fígado } \\
\hline Não & 218 & 52,8 & 1,00 & 0,8 \\
\hline Sim & 73 & 54,8 & $1,04(0,81-1,33)$ & \\
\hline \multicolumn{5}{|l|}{ Gema } \\
\hline Não & 139 & 59,0 & 1,00 & 0,06 \\
\hline Sim & 152 & 48,0 & $0,81(0,66-1,01)$ & \\
\hline \multicolumn{5}{|l|}{ Multimistura } \\
\hline Não & 283 & 55,1 & 1,00 & 0,02 \\
\hline Sim & 20 & 20,0 & $0,36(0,15-0,88)$ & \\
\hline \multicolumn{5}{|c|}{1 c.c. óleo prato } \\
\hline Não & 295 & 53,6 & 1,00 & 0,2 \\
\hline Sim & 8 & 25,0 & $0,47(0,14-1,56)$ & \\
\hline \multicolumn{5}{|l|}{ Papa grossa ${ }^{3}$} \\
\hline Não & 15 & 46,7 & 1,00 & 0,03 \\
\hline Sim & 24 & 87,5 & $1,87(1,06-3,31)$ & \\
\hline
\end{tabular}

${ }^{1}$ para menores de três anos somente / 'only for children younger than three years

${ }^{2}$ para todos os que não estavam sendo amamentados / ${ }^{2}$ for all children who were not being breastfed

${ }^{3}$ para os menores de oito meses de idade somente $/{ }^{3}$ only for children younger than eight months

desta doença decresce com a idade, sendo maior no segundo ano de vida, e a faixa mais ampla de idade das crianças deste estudo (menores de seis anos), era de se esperar uma menor prevalência em relação aos demais estudos. Tal achado provavelmente reflete as más condições de saúde das crianças moradoras nas áreas periféricas da cidade de Pelotas, tipicamente o grupo alvo da ação da Pastoral da Criança. De fato, a prevalência entre crianças de 12 a 23,9 meses de idade
(75,5\%; IC95\% 60,8\% - 86,2\%) foi superior à descrita por Osório et al. ${ }^{10}$ para essa mesma faixa etária, em Pernambuco (58,8\%; IC95\% $52,4 \%$ - 65,1\%); e, por Monteiro e Szarfarc ${ }^{5}$ na cidade de São Paulo (58,1\%; IC95\% 50,6\% - 65,2\%).

Acrescente-se a isso a acurácia do exame HemoCue para o diagnóstico de anemia. Em populações em que a concentração média de hemoglobina está abaixo de $11 \mathrm{~g} / \mathrm{dl}$ (abaixo de um desvio padrão da média de $12 \mathrm{~g} / \mathrm{dl}$ ), a 
Tabela 5 - Prevalência de anemia e Razão de Prevalências Bruta (com intervalo de confiança de 95\%) conforme indicadores antropométricos da criança. Pastoral da Criança, Pelotas, RS, 2003.

Table 5 - Prevalence of anemia and overall prevalence rates (with 95\% confidence interval) according to children's anthropometric indicators. Pastoral da Criança, Pelotas, RS, 2003.

\begin{tabular}{|c|c|c|c|c|}
\hline Característica & $\mathrm{N}(304)$ & $\begin{array}{l}\text { Prevalência } \\
\text { (\%) }\end{array}$ & $\begin{array}{l}\text { Razão de prevalência } \\
\text { bruta e IC95\% }\end{array}$ & P-valor \\
\hline \multicolumn{5}{|c|}{ Indicador peso/idade (quartis) } \\
\hline$\leq-1,37$ & 77 & 55,8 & 1,00 & 0,3 \\
\hline$-1,38--0,60$ & 76 & 60,5 & $1,08(0,83-1,42)$ & \\
\hline$-0,61-0,21$ & 69 & 46,4 & $0,83(0,60-1,15)$ & \\
\hline$\geq 0,22$ & 82 & 48,8 & $0,87(0,65-1,18)$ & \\
\hline \multicolumn{5}{|c|}{ Indicador peso/idade $<-2 \mathrm{DP} *$} \\
\hline Não & 274 & 51,8 & 1,00 & 0,4 \\
\hline Sim & 27 & 59,3 & $1,14(0,82-1,60)$ & \\
\hline \multicolumn{5}{|c|}{ Indicador altura/idade (quartis) } \\
\hline$\leq-1,11$ & 77 & 62,3 & 1,00 & 0,07 \\
\hline$-1,12--0,34$ & 79 & 51,9 & $0,83(0,63-1,10)$ & \\
\hline$-0,35-0,43$ & 68 & 57,4 & $0,92(0,70-1,20)$ & \\
\hline$\geq 0,44$ & 80 & 41,3 & $0,66(0,48-0,91)$ & \\
\hline \multicolumn{5}{|c|}{ Indicador altura/idade $<-2 \mathrm{DP} *$} \\
\hline Não & 270 & 51,5 & 1,00 & 0,3 \\
\hline Sim & 31 & 61,3 & $1,19(0,88-1,61)$ & \\
\hline \multicolumn{5}{|c|}{ Indicador peso/altura (quartis) } \\
\hline$\leq-1,14$ & 71 & 46,5 & 1,00 & 0,5 \\
\hline$-1,15--0,43$ & 78 & 53,8 & $1,16(0,84-1,60)$ & \\
\hline$-0,44-0,29$ & 74 & 59,5 & $1,28(0,94-1,75)$ & \\
\hline$\geq 0,30$ & 81 & 51,9 & $1,12(0,80-1,55)$ & \\
\hline \multicolumn{5}{|c|}{ Indicador peso/altura $<-2 \mathrm{DP} *$} \\
\hline Não & 293 & 52,9 & 1,00 & 0,5 \\
\hline Sim & 8 & 37,5 & $0,71(0,29-1,75)$ & \\
\hline
\end{tabular}

${ }^{1}$ para menores de três anos somente / 'only for children younger than three years

${ }^{2}$ para todos os que não estavam sendo amamentados / 2 for all children who were not being breastfed

${ }^{3}$ para os menores de oito meses de idade somente $/{ }^{3}$ only for children younger than eight months

*desvio padrão/ *standard deviation

prevalência estimada de anemia é enviesada no sentido de subestimar a verdadeira prevalência ${ }^{6}$. Estudos de validade do teste em sangue capilar mostraram um erro sistemático de $+0,5 \mathrm{~g} / \mathrm{dl}$ na leitura da concentração de hemoglobina, quando comparado com o padrão ouro ${ }^{7,11}$. A especificidade do teste para detectar anemia é adequada ( $>90 \%)$, mas a sensibilidade é relativamente baixa $(<80 \%)$. Corrigindo-se para esse erro, a prevalência de anemia na amostra passaria de $53,0 \%$ para 69,1\% (IC95\% 63,5\% - 74,2\%).
Na análise multivariada, apenas as variáveis idade da criança, classe social, presença de família numerosa, disponibilidade deágua no domicílio e peso ao nascer permaneceram significativamente associadas ao risco de anemia. O aumento de peso ao nascer, bem como a disponibilidade de água encanada dentro de casa apresentaram efeito protetor sobre o risco de ter anemia. Estes achados merecem investigações detalhadas em outros estudos. Viver em família numerosa, não ter pele branca e pertencer à classe social E, em 
Tabela 6 - Razões de prevalências ajustadas de anemia conforme modelo de análise hierárquico. Pastoral da Criança, Pelotas, RS, 2003.

Table 6 - Adjusted prevalence ratios for anemia according to the hierarchical analysis model. Pastoral da Criança, Pelotas, RS, 2003.

\begin{tabular}{lcc}
\hline Característica & Razão de prevalência ajustada e IC95\% & P-valor \\
\hline Classe social E & $1,37(1,20-1,58)$ & $<0,001$ \\
\hline Avó(s) vivem no domićílio & $1,51(1,12-2,04)$ & 0,007 \\
\hline Água encanada & $0,60(0,46-0,78)$ & $<0,001$ \\
\hline Peso ao nascer (quartis) & 1,00 & 0,005 \\
$1260-2799$ & $0,78(0,57-1,08)$ & \\
$2800-3169$ & $0,94(0,71-1,24)$ & \\
$3170-3499$ & $0,58(0,43-0,79)$ & \\
$3500-4860$ & & \\
\hline Idade da criança & $3,36(1,59-7,01)$ & \\
$<1212-23,9$ & $2,90(1,17-7,21)$ & \\
$24-35,9$ & $2,63(1,23-5,64)$ & \\
$36-47,9$ & $1,68(0,75-3,76)$ & \\
$48-59,9$ & $1,43(0,70-2,92) 1,00$ & 0,03 \\
$60-71,9$ & $1,32(1,02-1,69)$ & \\
\hline Cor da pele não branca & &
\end{tabular}

comparação à classe social $\mathrm{D}$, ao contrário, aumentaram o risco de anemia.

A maior prevalência de anemia observada entre as crianças de famílias da classe social E, em comparação com as de famílias da classe D, é consistente com os achados de Monteiro e Szarfarc ${ }^{5}$ em São Paulo, Neumann et al. ${ }^{8}$ em Criciúma, e Silva et al. ${ }^{15}$ em Porto Alegre. As maiores prevalências de anemia associadas aos menores pesos de nascimento reforçam as evidências de que a anemia na infância resulte de um aporte deficiente de ferro ainda durante a gestação. Em uma população em que $10 \%$ dos menores de seis anos estão abaixo de -2 desvios padrões para os escores peso/idade e altura/idade, a ausência de associação com os indicadores antropométricos possivelmentese deve ao baixo teor de ferro, acrescido de baixa biodisponibilidade desse mineral na dieta habitual das crianças estudadas, indistintamente do peso e comprimento que tenham alcançado.Afalta de contrastena amostra inviabilizaria a possibilidade de se detectar associação.

Finalmente, vale destacar que apenas $7,5 \%$ dos casos de anemia detectados pelo estudo eram do conhecimento da mãe (sen- sibilidade). A taxa de informações falso-positivas foi também muito baixa, devido a uma especificidade de $91,9 \%$. O valor preditivo positivo da informação materna foi muito baixo (50,5\%) e, entre as que afirmavam que não sabiam se a criança tinha anemia, a probabilidade de exame positivo foi de 51,7\%. Apesar da alta prevalência, a baixa sensibilidade da informação materna quanto ao diagnóstico atual de anemia na criança e a pequena proporção de crianças em uso de sulfato ferroso (10,8\%) parecem indicar que, na população estudada, a anemia infantil é um problema de saúde altamente subdiagnosticado. Este subdiagnóstico sugere que o problema da anemia na infância não tem recebido a devida ênfase na agenda da atenção primária à saúde.

O presente estudo torna evidente a necessidade de concentrar esforços na melhoria da qualidade do pré-natal, prevenindo os fatores associados a menores pesos de nascimento e suplementando profilaticamente a mãe com sais de ferro. Esta suplementação poderá contribuir para um maior aporte do mineral pelo feto durante a vida intra-uterina. 
Além disso, a educação da mãe desde a gestação em prol do aleitamento exclusivo até os seis meses de idade e a utilização de alimentos complementares nutricionalmente adequados poderão contribuir para a proteção de futuras gerações quanto a essa carência nutricional.

\section{Agradecimentos}

Os autores agradecem o apoio financeiro da Fundação Itaú Social, através da Pastoral da Criança, aos coordenadores e às Líderes da Pastoral em Pelotas e a todas as mães e crianças que tornaram possível a realização desse estudo.

\section{Referências}

1. Araújo RL, Araújo MBDG, Sieiro RO, Machado RD, Leite BV. Diagnóstico de hipovitaminose A e anemia nutricional. Estudo realizado na população do Vale do Jequitinhonha, Minas Gerais. Rev Bras Med 1986; 43(8): 225-8.

2. Baker SJ. Nutritional anemia: a major controlable public health problem. Bull World Health Organ 1978; 56: 659-75.

3. Ferreira MR, Souza W, Perez EP, Lapa T, Carvalho AB, Furtado A et al. Intestinal helminthiasis and anemia in youngsters from Matriz da Luz, district of São Lourenço da Mata, state of Pernambuco, Brazil. Mem Inst Oswaldo Cruz 1998; 93(3): 289-93.

4. Hallberg L, Rossander-Hulten L, Brune M, Gleerup A. Bioavailability in man of iron in human milk and cow's milk in relation to their calcium contents. Pediatric Res 1992; 31: 524-7.

5. Monteiro CA, Sczarfarc SC. Estudo das condições de saúde das crianças no Município de São Paulo, SP (Brasil), 1984-1985. V - Anemia. Rev Saúde Pública 1987; 21(3):255-60.

6. Morris SS, Ruel MT, Coehn RJ, Dewey KG, Brière B, Hassan M. Precision, accuracy, and reliability of hemoglobin assessment with use of capillary blood. Am J Clin Nutr 1999; 69:1243-8.

7. Neufeld L, Garcia-Guerra A, Sánchez-Francia D, Newton-Sánchez O, Ramirez-Villalobos MD, RiveraDommarco J. Hemoglobin measured by HemoCue and a reference method in venous and capillary blood: a validation study. Salud Publica Mex 2002; 44(3): 21927.

8. Neumann NA, Tanaka OY, Szarfarc SC, Guimarães PRV, Victora CG. Prevalência e fatores de risco para anemia no sul do Brasil. Rev Saúde Pública 2000; 34(1): 56-63.
9. Oliveira RS, Diniz AS, Benigna MJC, Miranda-Silva SM, Lola MM, Gonçalves MC et al. Magnitude, distribuição espacial e tendência da anemia em pré-escolares da Paraíba. Rev Saúde Pública 2002; 36(1): 26-32.

10. Osório MM, Lira PIC, Batista-Filho M, Ashworth A. Prevalence of anemia in children 6-59 months old in the state of Pernambuco, Brazil. Pan Am J Public Health 2001; 10(2):101-7.

11. Prakash S, Kapil U, Singh G, Dwivedi SN, Tandon M. Utility of HemoCue in estimation of hemoglobin against standard blood cell counter method. J Assoc Physicians India 1999; 47(10):995-7.

12. Santos I, Minten G, Cesar JA, Valle N. Treinamento das Líderes da Pastoral da Criança em aconselhamento nutricional e anemia entre menores de seis anos de idade. Departamento de Medicina Social da Universidade Federal de Pelotas; 2003.

13. Schieri R, Szarfarc SC, Monteiro CA. Relação entre dieta e ocorrência de anemia ferropriva em crianças. $J$ Pediatr (Rio J) 1988; 64(5):169-74.

14. Sigulen DM, Tudisco ES, Goldemberg P, Athaide MM, Vaisman E. Anemia ferropriva em crianças do município de São Paulo. Rev Saúde Pública 1978; 12(2): $168-78$.

15. Silva LSM, Giugliani ERJ, Aerts DRGC. Prevalência e determinantes de anemia em crianças de Porto Alegre, RS, Brasil. Rev Saúde Pública 2001; 35(1): 66-73.

16. Tsuyuoca R, Bailey JW, Guimaraes AMAN. Anemia and intestinal parasitic infection in primay school children in Aracaju, Sergipe, Brazil. Cad Saúde Pública 1999; 15(2): $413-21$.

recebido em: 26/08/2004 versão final apresentada em: 25/11/2004 aprovado em: 06/12/2004 